\title{
Global Identifiability of Complex Models, Constructed from Simple Submodels
}

Markus Gerdin, Torkel Glad, Lennart Ljung

Division of Automatic Control

E-mail: gerdin@isy.liu.se, torkel@isy.liu.se,

ljung@isy.liu.se

14th June 2007

Report no.: LiTH-ISY-R-2783

Accepted for publication in Modeling, Estimation and Control: Festschrift in honor of Giorgio Picci on the occasion of his sixty-fifth Birthday, Springer, 2007

Address:

Department of Electrical Engineering

Linköpings universitet

SE-581 83 Linköping, Sweden

WWW: http://WwW. control.isy.liu.se

AUTOMATIC CONTROL REGLERTEKNIK LINKÖPINGS UNIVERSITET

Technical reports from the Automatic Control group in Linköping are available from http://www. control.isy.liu.se/publications. 


\begin{abstract}
It is a typical situation in modern modeling that a total model is built up from simpler submodels, or modules, for example residing in a model library. The total model could be quite complex, while the modules are well understood and analysed. A procedure to decide global parameter identifiability for such a collection of model equations of differential-algebraic nature is suggested. It is shown how to make use of the natural modularization of the model structure. Basically, global identifiability is obtained if and only if each module is identifiable, and the connecting signals can be retrieved from the external signals, without knowledge of the values of the parameters.
\end{abstract}

Keywords: identification, identifiability 


\title{
Global Identifiability of Complex Models, Constructed from Simple Submodels ${ }^{\star}$
}

\author{
Markus Gerdin $^{1}$, Torkel Glad ${ }^{2}$ and Lennart Ljung ${ }^{2}$ \\ 1 NIRA Dynamics AB, Gothenburg, Sweden, markus.gerdin@gmail.com \\ 2 Linköping University, S-58183 Linköping Sweden, torkel, 1jung@isy.liu.se
}

To Giorgio, with admiration, on the occasion of his 65th birthday

\begin{abstract}
It is a typical situation in modern modeling that a total model is built up from simpler submodels, or modules, for example residing in a model library. The total model could be quite complex, while the modules are well understood and analysed. A procedure to decide global parameter identifiability for such a collection of model equations of differential-algebraic nature is suggested. It is shown how to make use of the natural modularization of the model structure. Basically, global identifiability is obtained if and only if each module is identifiable, and the connecting signals can be retrieved from the external signals, without knowledge of the values of the parameters.
\end{abstract}

\section{Introduction}

Identifiability is a crucial concept in System Identification. It concerns the question of whether the parameters in a model structure can be uniquely retrieved from input-output data. Clearly, being able to assess the identifiability of a structure beforehand, without going through all the estimation labor would be a very helpful technique.

The literature on identifiability and techniques to check identifiability is extensive, see, e.g. [3], Chapter 4, [7, 8], [4]. Identifiability of linear black-box models in terms of canonical forms etc, is basically a solved problem. However

\footnotetext{
* This work has been supported by the Swedish Foundation for Strategic Research (SSF) through VISIMOD and ECSEL and by the Swedish Research Council (VR) which is gratefully acknowledged.
} 
even for linear models physically parameterized structures form a highly nontrivial challenge, see e.g. [5], and there are no efficient techniques other than in certain subclasses of problems. The idea of [4] to use differential algebra leads to a well defined algorithm for quite general structures, but it suffers from too high computational complexity in many realistic cases.

In this contribution we shall consider a common situation in defining model structures: Suppose that the model is made of from several interconnected modules. Each of these modules are simple and have well defined identifiability properties, in case they are encountered on their own. The modules are interconnected in well defined ways, but the interconnecting signals are not necessarily measured. The question is then how the identifiability of the total model can be studied in terms of the module properties and their interconnections. This way of dealing with model structures is typical in modular-based (or object-oriented) modeling environments, such as ModeLICA, see e.g. [1]. A more complete discussion on identifiablity of modular models described by algebraic differential equations (DAEs) is given in [2].

\section{The Problem}

Consider a model structure built up from a collection of sub-models, or modules, $M_{k}, k=1, \ldots, m$. Each module is a model that describes the relationship between a number of connecting signals $w_{k, i}(t), i=1, \ldots, n_{k}$. This relationship involves also a number of internal signal $\ell_{k, i}(t), 1=1, \ldots, p_{k}$ and a number of parameters $\theta_{k, i}, i=1, \ldots, q_{k}$. The relations may be expressed as a collection of algebraic or differential equations. With $p$ denoting the differentiation operator, the model for module $M_{k}$ can be written

$$
\begin{gathered}
f_{k, i}\left(\ell_{k, 1}(t), \ldots, \ell_{k, p_{k}}(t), w_{k, 1}(t), \ldots, w_{k, n_{k}}(t),\right. \\
\left.\ldots, \theta_{k, 1}, \ldots \theta_{k, q_{k}}, p\right)=0, i=1, \ldots, r_{k}
\end{gathered}
$$

With obvious "vectorization" of $\ell_{k, \cdot}, w_{k, .}, \theta_{k,}$. and $f_{k,}$. we will write the above equation as

$$
f_{k}\left(\ell_{k}(t), w_{k}(t), \theta_{k}, p\right)=0
$$

The modules are then connected by describing how the connecting signals $w_{k}$ interconnect and relate to globally external signals $z(t)$ ("inputs" and "outputs")

$$
g\left(z(t), w_{1}(t), \ldots, w_{m}(t)\right)=0
$$

Typically the inputs and outputs will be equal to some of the connecting signals $w_{k, i}$. The distinction between inputs and outputs is immaterial for the present discussion. See Figure 1. 
As a simple case, the reader may picture $M_{k}$ as resistors, capacitors and inductors, with well known relationships (1a) between voltage drops, currents $\left(\ell_{k}(t), w_{k}(t)\right)$ and component parameters $\theta_{k}$ (resistance, capacitance, inductance). An arbitrary RLC-circuit can then be defined by the interconnections (1b) between voltages and currents $w_{k}(t)$ following Kirchhoff's laws.

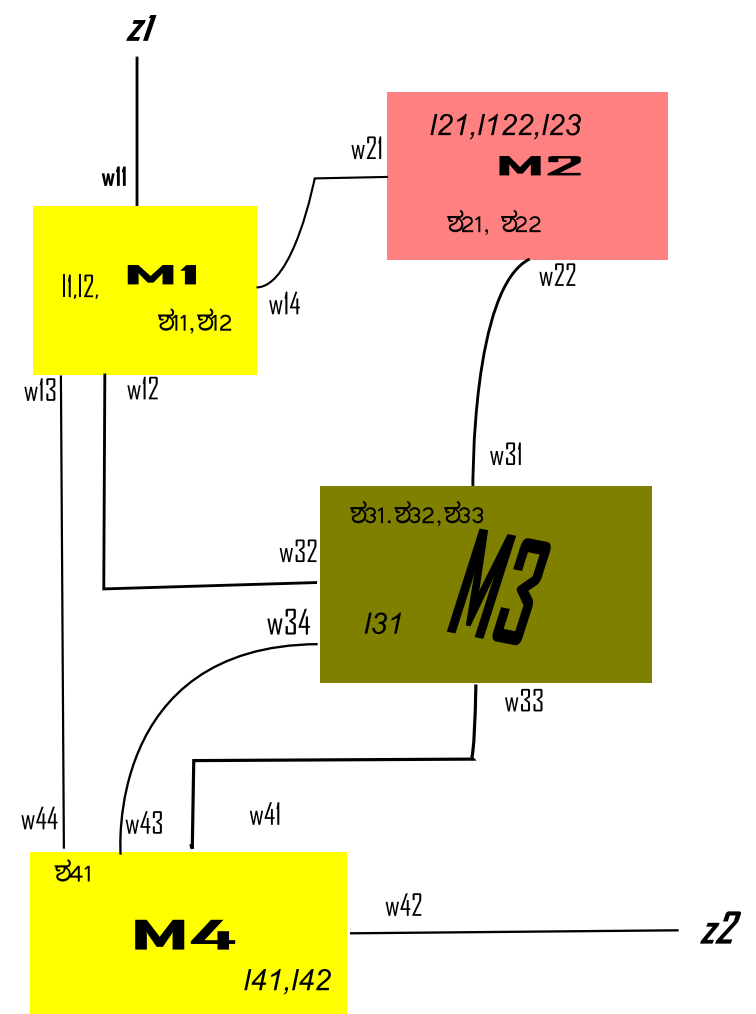

Fig. 1. Interconnected modules of submodels. $w_{k, i}$ is the $i$ :th external connecting signal in module $k$ and $z$ are the global external signals. $\ell_{k, i}$ and $\theta_{k, i}$ (with a strange font) are internal signals and parameters in the modules.

Now, the total model is given as (1) with $z(t)$ as external signals, also depicted in Figure 1. The identifiability question for this total model is

- Given the external signals $z(t), t \in T$, is it possible to uniquely determine all the parameters $\theta_{k}, k=1, m$ ? That is, can the equations (1) be satisfied for different parameter values for a given $z(t), t \in T$ ? 
If it indeed is possible to uniquely retrieve the parameter values, we say that the model is globally identifiable for the given external signal. (See, e.g. [4] and [2] for more strict definitions.)

The following result is plausible

Result 1 The model (1) is globally identifiable if and only if

a) The external signals $w_{k}(t)$ can be uniquely retrieved from $z(t)$ and (1), without knowledge of the parameter values $\theta_{k}$.

b) Each module $M_{k}$ is globally identifiable for retrieved external signal $w_{k}(t)$

A formal version of this result will be proved in the course of this contribution, but the result is not difficult to appreciate and understand intuitively. Clearly a) and b) will imply global identifiability, since with retrieved interconnecting signals $w_{k}$ identifiability of the modules will guarantee that all parameters can be found uniquely. Conversely, if a module is not identifiable with known signals $w_{k}$ it is clear that its parameters cannot be found when the signals are not known (or reconstructed). It is perhaps somewhat less obvious to realize that $w_{k}$ can be uniquely retrieved for a globally identifiably model structure. However, the argument is as follows: Suppose the model structure is well defined, so that the model can be simulated for any set of parameter values. Then with the globally identified parameters inserted and the (input part of the) external signals $z(t)$ well defined, all interconnecting signals $w_{k}$ will be generated in a unique way by such a simulation.

\section{A Simple Example of Interconnected Modules}

To illustrate the role of the interconnecting signals, let us consider an almost trivial example. Take a module as an integrator with unknown gain. The input to the integrator is $w_{1}$ and its output is $w_{2}$ :

$$
\dot{w}_{2}(t)=\theta_{1} w_{1}(t)
$$

Clearly this module is globally identifiable with known external signals $w_{1}, w_{2}$. Let us now cascade two such modules:

$$
\dot{w}_{4}(t)=\theta_{2} w_{3}(t)
$$

For the cascaded system, we have input $z_{1}(t)=w_{1}(t)$ and output $z_{2}(t)=$ $w_{4}(t)$ so the interconnecting equations are

$$
\begin{aligned}
z_{1}(t) & =w_{1}(t) \\
w_{3}(t) & =w_{2}(t) \\
z_{2}(t) & =w_{4}(t)
\end{aligned}
$$

The model structure obtained by cascading these two identifiable modules is however not identifiable: From measuring only $z(t)$ we can find out the product 
$\theta_{1} \cdot \theta_{2}$ but not the individual values. In view of Result 1 in the previous section, this must mean that the interconnecting signal $w_{3}=w_{2}$ cannot be uniquely retrieved from $z(t)$, even knowing that the signals relate via integrators. In fact, we will know that $w_{2}$ is proportional to $\dot{z}_{2}(t)$ but have no way to find the coefficient of proportionality.

Now, if, say $\theta_{1}$ is known to be $\theta_{1}=1$, the global structure should be identifiable. That means that $w_{2}(t)$ can be uniquely retrieved, but it is instructive to realize that it has to be done with care. Knowing the double integrator structure and the input $z_{1}(t)$ we can only conclude that

$$
w_{2}(t)=\int z_{1}(s) d s+\text { Constant }
$$

where the constant is due to initial conditions. So $w_{2}$ cannot be uniquely retrieved. However, knowing also $z_{2}(t)$ gives the relation

$$
\theta_{2} w_{2}(t)=\dot{z}_{2}(t)
$$

From this we may obtain (differentiate (4), use $\dot{w}_{2}=z_{1}$ and eliminate $\theta_{2}$ from the obtained two equations):

$$
\ddot{z}_{2}(t) w_{2}(t)=z_{1}(t) \dot{z}_{2}(t)
$$

so, as long as $\ddot{z}_{2}(t)$ is nonzero we can find $w_{2}(t)$ uniquely. Here are no unknown constants nor initial conditions involved. The simple example also shows that some care must be exercised when examining the retrieval of the interconnecting signals. The condition that $\ddot{z}_{2}$ is nonzero means that $z_{1}$ and $\theta_{1} \dot{\theta}_{2}$ must be nonzero. This is an example of an excitation condition on the (input) signals, and particular parameter values that typically is required to assure identifiability.

\section{Preliminary Considerations and Tools}

Our goal is to use Result 1 to study identifiability of complex model structures (1) in terms of identifiability of the modules $M_{k}$. In many applications these modules will be standardized in model libraries so the identifiability of these can be examined once and for all. The crux then is to establish condition a) in the Result, that the interconnecting signals can be uniquely retrieved from the external signals $z(t)$, and the model equations without knowledge of the parameter values. An obvious way is to find a parameter-free relation, such as (5) from which $w$ can be determined from $z$. How can that be done?

For that we will use the tools of differential algebra, [6] as applied to identifiability in [4]. In short, the idea is that the original set of model equations can be transformed to a new set by differentiating, and performing simple algebraic operations. Under certain conditions the solutions to the new equation 
set will be identical to the solutions of the original one. So the identifiability analysis can be applied to the new set instead. It is then a matter to let the new set be as well suited as possible for such analysis.

Remark: Strictly speaking the formal result is limited to the case that the $f_{k}$ are polynomial expressions. However, more general cases can also be handled. Such a case is given in Example 3 below.

More specifically, consider a module (1a):

$$
f_{k}\left(\ell_{k}(t), w_{k}(t), \theta_{k}, p\right)=0
$$

where $f_{k}$ is a vector, so that the expression covers several model equations. By manipulating these equations with differential algebraic tools, they can be transformed to the following set

$$
\begin{aligned}
& A_{k, 1}\left(w_{k}, p\right), \ldots, A_{k, n_{A_{k}}}\left(w_{k}, p\right), \\
& B_{k, 1}\left(w_{k}, \theta_{k, 1}, p\right), B_{k, 2}\left(w, \theta_{k, 1}, \theta_{k, 2}, p\right), \\
& B_{k, n_{\theta_{k}}}\left(w_{k}, \theta_{k, 1}, \theta_{k, 2}, \ldots, \theta_{k, n_{\theta_{k}}}, p\right), \\
& C_{k, 1}\left(w_{k}, \theta_{k}, \ell_{k}, p\right), \ldots, C_{k, n_{l_{k}}}\left(w_{k}, \theta_{k}, \ell_{k}, p\right) .
\end{aligned}
$$

The $A$-equations are then just relationships between (derivatives of) the interconnecting signals $w_{k}$, not involving the internal variables $\ell_{k}$ nor the parameters. The $B$-equations will reveal whether the parameters $\theta_{k}$ are identifiable. The main result in [4] is that the module $M_{k}$ is globally identifiable if and only if the $B$-equations have a linear regression form:

$$
B_{k}=P_{k}\left(w_{k}, p\right) \theta_{k}-Q_{k}\left(w_{k}, p\right),
$$

The excitation condition (see the end of Section 3) on $w_{k}$ is then that $P_{k}$ is invertible.

Example 1. Capacitor: Consider a capacitor described by the voltage drop $w_{1}$, current $w_{2}$ and capacitance $\theta_{1}$. It is then described by (1a) with

$$
f_{1}=\left(\begin{array}{c}
\theta_{1} \dot{w}_{1}-w_{2} \\
\dot{\theta}_{1}
\end{array}\right) \text {. }
$$

If we consider only situations where $\dot{w}_{1} \neq 0$ we get the following series of equivalences.

$$
\begin{aligned}
& \theta_{1} \dot{w}_{1}-w_{2}=0, \quad \dot{\theta}_{1}=0, \quad \dot{w}_{1} \neq 0 \\
& \Leftrightarrow \\
& \theta_{1} \dot{w}_{1}-w_{2}=0, \quad \theta_{1} \ddot{w}_{1}-\dot{w}_{2}=0, \quad \dot{w}_{1} \neq 0 \\
& \Leftrightarrow \\
& \theta_{1} \dot{w}_{1}-w_{2}=0, \quad \theta_{1} \dot{w}_{1} \ddot{w}_{1}-\dot{w}_{1} \dot{w}_{2}=0, \quad \dot{w}_{1} \neq 0 \\
& \Leftrightarrow \\
& \theta_{1} \dot{w}_{1}-w_{2}=0, \quad w_{2} \ddot{w}_{1}-\dot{w}_{1} \dot{w}_{2}=0, \quad \dot{w}_{1} \neq 0
\end{aligned}
$$


With the notation (7) we thus have

$$
\begin{aligned}
& A_{1,1}=w_{2} \ddot{w}_{1}-\dot{w}_{1} \dot{w}_{2} \\
& B_{1,1}=\theta_{1} \dot{w}_{1}-w_{2}
\end{aligned}
$$

The capacitor is thus globally identifiable, provided $\dot{w}_{1} \neq 0$.

This gives an idea of how to handle condition a) in Result 1: If we work with previously analyzed modules $M_{k}$ in a model library, we have already their $A$-equations, (7a). We can dispense with the remaining $B$ and $C$-equations. For global identifiability of the whole model we need to consider the total collection of $A$-equations together with the connecting equations (1b) and check whether all the interconnecting signals $w$ can be retrieved from this set of equations,

$$
\begin{aligned}
& A_{1,1}\left(w_{1}, p\right), \ldots, A_{m, n_{A_{m}}}\left(w_{m}, p\right) \\
& g(z(t), w(t))=0
\end{aligned}
$$

knowing the external variables $z(t)$. Here $(12 \mathrm{~b})$ is a more compact way of writing (1b).

\section{Identifiability Analysis}

In this section we shall illustrate how to use Result 1 for identifiability analysis based on submodels in a model libraray. We will use a minimal model library consisting of a resistor model, an inductor model, and a capacitor model. Note that these components have corresponding components for example within mechanics and fluid systems. Bond graphs make full use of such analogies: it follows that our simple model library is quite representative of more general cases. A capacitor model was described in Example 1. Similarly, we have

Example 2. Inductor: Next consider an inductor where $w_{2}$ is the current, $w_{1}$ the voltage and $\theta_{1}$ the inductance. It is described by

$$
\theta_{1} \dot{w}_{2}=w_{1}, \quad \dot{\theta}_{1}=0
$$

Calculations similar to those of the previous example show that this is equivalent to

$$
\theta_{1} \dot{w}_{2}=w_{1}, \quad \ddot{w}_{2} w_{1}=\dot{w}_{2} \dot{w}_{1}
$$

provided $\dot{w}_{2} \neq 0$.

As discussed earlier, the transformation to (7) can always be performed for polynomial DAE. To show that calculations of this type in some cases also can be done for non-polynomial models, we consider a nonlinear resistor where the voltage drop is given by an arbitrary function. 
Example 3. Nonlinear Resistor: Consider a nonlinear resistor with the equation

$$
w_{1}=R\left(w_{2}, \theta_{1}\right)
$$

where it is assumed that the parameter $\theta_{1}$ can be uniquely solved from (15) if the voltage $w_{1}$ and the current $w_{2}$ are known, so that

$$
\theta_{1}=\phi\left(w_{1}, w_{2}\right)
$$

Differentiating (15) once with respect to time and inserting (16) gives

$$
\dot{w}_{1}=R_{w_{2}}\left(w_{2}, \phi\left(w_{1}, w_{2}\right)\right) \dot{w}_{2}
$$

which is a relation between the external variables $w_{1}$ and $w_{2}$. We use the notation $R_{x}$ for the partial derivative of $R$ with respect to the variables $x$. In the special case with a linear resistor, where $R=\theta_{1} \cdot w_{2}$, this reduces to

$$
\begin{aligned}
\dot{w}_{1} & =\frac{w_{1}}{w_{2}} \dot{w}_{2} \\
\Leftrightarrow w_{2} \dot{w}_{1} & =w_{1} \dot{w}_{2}
\end{aligned}
$$

(assuming $\left.w_{2} \neq 0\right)$.

We shall here examine the identifiability of different connections of the components.

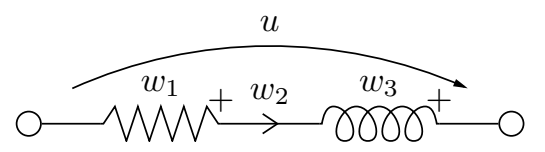

Fig. 2. A resistor and an inductor connected in series.

Example 4. Consider a nonlinear resistor and an inductor connected in series where the current $w_{2}=f$ and total voltage $u$ are measured as shown in Fig. 2 . Denote the voltage over the resistor with $w_{1}$ and the voltage over the inductor with $w_{3}$. Using Examples 2 and 3 we get the equations

$$
\begin{aligned}
\dot{w}_{1} & =R_{w_{2}}\left(w_{2}, \phi\left(w_{1}, w_{2}\right)\right) \dot{w}_{2} \\
\ddot{w}_{2} w_{3} & =\dot{w}_{2} \dot{w}_{3}
\end{aligned}
$$

for the components and the equation

$$
w_{1}+w_{3}=u
$$

for the connections. Differentiating the last equation once gives

$$
\dot{w}_{1}+\dot{w}_{3}=\dot{u}
$$


The system of equations (19) (with $w_{1}, \dot{w}_{1}, w_{3}$, and $\dot{w}_{3}$ as unknowns) has the Jacobian

$$
\left(\begin{array}{cccc}
-R_{w_{2}, w_{1}} \dot{w}_{2} & 1 & 0 & 0 \\
0 & 0 & \ddot{w}_{2} & -\dot{w}_{2} \\
1 & 0 & 1 & 0 \\
0 & 1 & 0 & 1
\end{array}\right)
$$

where

$$
R_{w_{2}, w_{1}}=\frac{\partial}{\partial w_{1}}\left(R_{w_{2}}\left(w_{2}, \phi\left(w_{1}, w_{2}\right)\right)\right) .
$$

The Jacobian has the determinant $-R_{w_{2}, w_{1}} \cdot \dot{w}_{2}^{2}+\ddot{w}_{2}$, so the system of equations is solvable for most values of the external variables. This means that the system is identifiable.

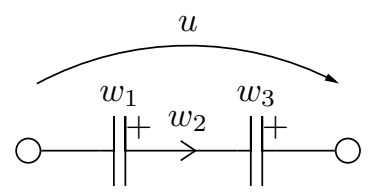

Fig. 3. Two capacitors connected in series.

Example 5. Now consider two capacitors connected in series where the current $w_{2}=f$ and total voltage $u$ are measured as shown in Fig. 3. Denote the voltages over the capacitors with $w_{1}$ and $w_{3}$ respectively. Using Example 1 we get the equations

$$
\begin{aligned}
& w_{2} \ddot{w}_{1}=\dot{w}_{1} \dot{w}_{2} \\
& w_{2} \ddot{w}_{3}=\dot{w}_{3} \dot{w}_{2}
\end{aligned}
$$

for the components. The connection is described by the equation

$$
w_{1}+w_{3}=u .
$$

These equations directly give that if

$$
\begin{aligned}
& w_{1}(t)=\phi_{1}(t) \\
& w_{3}(t)=\phi_{3}(t)
\end{aligned}
$$

is a solution, then so are all functions of the form

$$
\begin{aligned}
& w_{1}(t)=(1+\lambda) \phi_{1}(t) \\
& w_{3}(t)=\phi_{3}(t)-\lambda \phi_{1}(t)
\end{aligned}
$$

for scalar $\lambda$. Since (11b) implies that the capacitance is an injective function (i.e. no two arguments give the same function value) of the derivative of the voltage, this shows that the system is not identifiable. 


\section{A Formal Theorem on Identifiability from Sub-models}

We shall in this section prove a formal version of Result 1.

Consider a model structure consisting of $m$ interconnected modules $M_{k}$, (1a). Assume that the different modules do not have common parameters, and that the differential algebraic equations (1) are polynomial in their arguments. Assume also that all modules are identifiable if their connecting variables $w_{i}$ are measured. This means, that given measurements of

$$
w_{i} \quad i=1, \ldots, m
$$

the unknown parameters $\theta$ can be computed uniquely from the $B$-polynomials in $(7 \mathrm{~b})$. When examining identifiability of the connected system it is not a big restriction to assume that the individual components are identifiable since information is removed when not all $w_{i}$ are measured.

When the components have been connected, the only knowledge available about the $w_{i}$ is the $A$-polynomials in (7a) and the equation

$g(z(t), w(t))=0$.The connected system is thus identifiable if the $w_{i}$ can be computed from (12):

$$
\begin{aligned}
& A_{i j}\left(w_{i}(t), p\right)=0 \quad\left\{\begin{array}{l}
i=1, \ldots, m \\
j=1, \ldots, n_{A_{i}}
\end{array}\right. \\
& g(z(t), w(t))=0 .
\end{aligned}
$$

Note that this means that all $w(t)$ are algebraic variables (not differential), so that no initial conditions can be specified for any component of $w(t)$. If, on the other hand, there are several solutions to the equations (27) then these different solutions can be inserted into the $B$ polynomials, so there are also several possible parameter values. In this case the connected system is therefore not identifiable. Note again that measured inputs and outputs lead to equations of the form $w_{i}(t)=u(t)$, where the function $u$ is included in the time-variability of $g$.

The result is formalized in the following theorems. Note that the distinction between global and local identifiability was not discussed above, but this will be done below.

\subsection{Global Identifiability}

Global identifiability means that there is a unique solution to the identification problem, given that the measurements are informative enough. For a subsystem (1a) that can be rewritten in the form (7) global identifiability means that the $B_{i, j}$ can be solved uniquely to give the $\theta_{i, j}$. In other words there exist functions $\psi$, that can in principle be calculated from the $B_{i, j}$, such that

$$
\theta_{i}=\psi_{i}\left(w_{i}, p\right)
$$

We then have the following formal result on identifiability. 
Theorem 1 Consider a modular model structure where the modules (1a) are globally identifiable and thus can be described in the form (28). A sufficient condition for the total model structure to be globally identifiable is that (27) can be solved uniquely for the $w_{i}$. If all the functions $\psi_{i}$ of (28) are injective then this condition is also necessary.

Proof: If (27) gives a global solution for $w(t)$, then this solution can be inserted into the $B$ polynomials to give a global solution for $\theta$ since the components are globally identifiable. The connected system is thus globally identifiable. If there are several solutions for $w_{i}$ and the functions $\psi_{i}$ of (28) are injective, then there are also several solutions for $\theta$, so the system is not globally identifiable since the identification problem has more than one solution.

\subsection{Local Identifiability}

Local identifiability of a model structure means that locally there is a unique solutions to the identification problem, but globally there may be more than one solution. This means that the description (28) is valid only locally. We get the following result on local identifiability.

Theorem 2 Consider a modular model structure where the modules (1a) are locally identifiable and thus can be locally described in the form (28). A sufficient condition for the total model to be locally identifiable is that (27) can be solved locally uniquely for the $w_{i}$. If all the functions $\psi_{i}$ of (28) are locally injective then this condition is also necessary.

Proof: If (27) gives a locally unique solution for $w(t)$, then this solution can be inserted into the $B$ polynomials to give a local solution for $\theta$ since the components are locally identifiable. The connected system is thus locally identifiable. If there locally are several solutions for $w_{i}$ and the functions $\psi_{i}$ of (28) are injective, then there are also several local solutions for $\theta$, so the system is not locally identifiable since the identification problem locally has more than one solution.

\section{Conclusions}

This paper has shown how a modular structure of a large model can be used to simplify examination of identifiability. For modules in model libraries, the transformation to the form (7) is computed once and for all and stored with the module. This makes it possible to only consider a smaller number of equations when examining identifiability for a model composed of such modules. Although the method described in this paper may suffer from high computational complexity (depending, among other things, on the method selected for deciding the number of solutions for (12)), it can make the situation much better than when trying to use to use the differential-algebra approach described in [4] on a complete model. 
The technique could be included in tools for object-oriented modeling such as Dymola and Openmodelica. Preferably, this could be part of a complete set of system identification routines linked to the modeling software. The identification routines could either be included directly in the modeling software, or as external software that interacts with the modeling software.

Future work could include to examine if it is possible to make the method fully automatic, so that it can be included in modeling tools and to examine if other system analysis or design methods can benefit from the modularized structure in object-oriented models. It could also be interesting to examine the case when several components share the same parameter. This could occur for example if the different parts of the system are affected by environmental parameters such as temperature and fluid constants.

\section{References}

1. Peter Fritzson. Priciples of Object-Oriented Modeling and Simulation with Modelica 2.1. Wiley IEEE, New York, 2004.

2. Markus Gerdin. Identification and Estimation for Models Described by Differential-Algebraic Equations. PhD thesis, Linköping University, Linköping, Sweden, 2006. Dissertation No 1046.

3. L. Ljung. System Identification - Theory for the User. Prentice-Hall, Upper Saddle River, N.J., 2nd edition, 1999.

4. Lennart Ljung and Torkel Glad. On global identifiability of arbitrary model parameterizations. Automatica, 30(2):pp 265-276, Feb 1994.

5. P. Parrilo and Lennart Ljung. Initialization of physical parameter estimates. In P. van der Hof, B. Wahlberg, and S. Weiland, editors, Proc. 13th IFAC Symposium on System Identification, pages 1524 - 1529, Rotterdam, The Netherlands, Aug 2003.

6. J.F. Ritt. Differential Algebra. American Mathematical Society, Providence, R.I., 1950.

7. E. Walter. Identification of State Space Models. Springer Verlag, Berlin, 1982.

8. E. Walter, editor. Identifiability of Parametric Models. Pergamon Press, Oxford, 1987. 


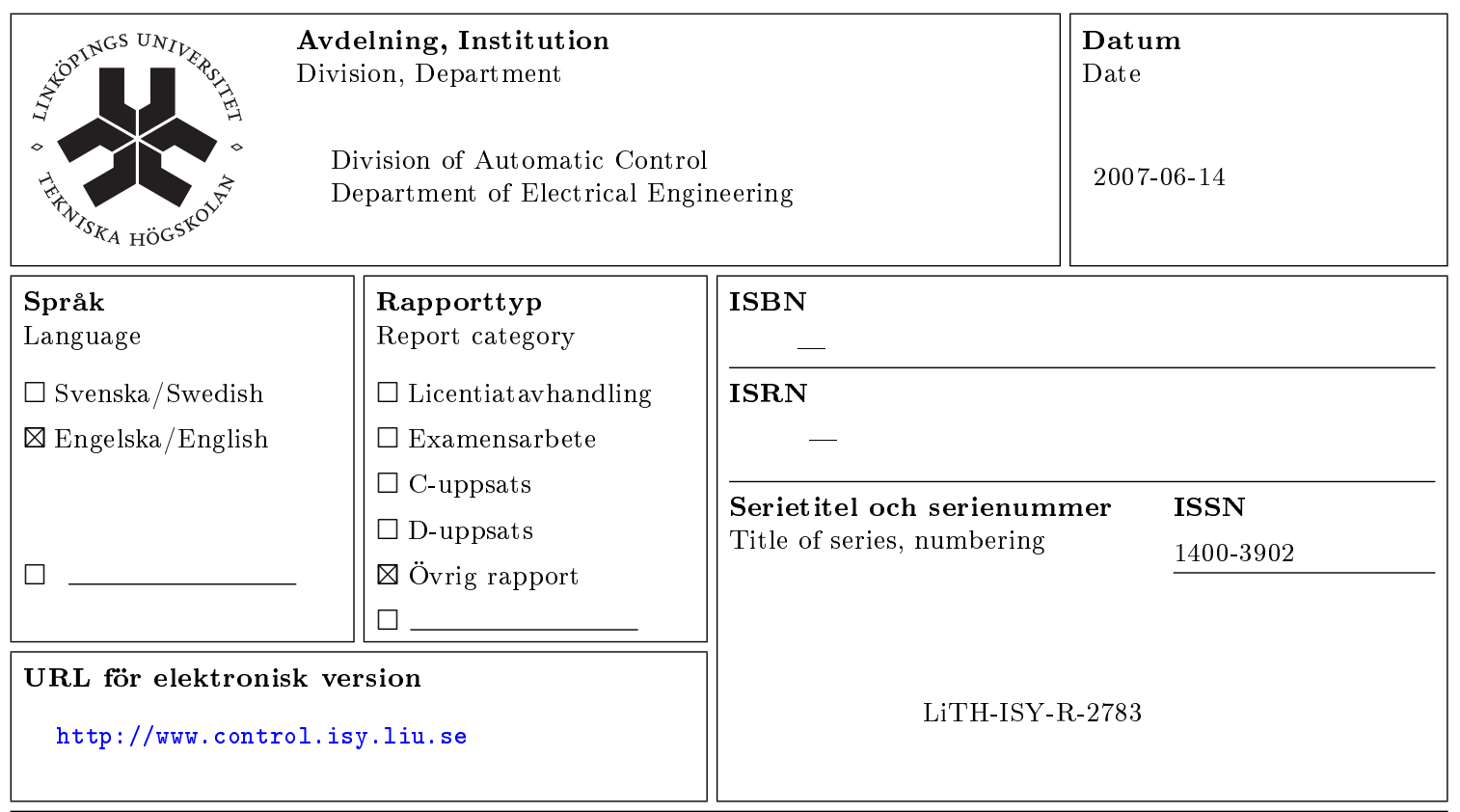

Titel Global Identifiability of Complex Models, Constructed from Simple Submodels

Title

Författare Markus Gerdin, Torkel Glad, Lennart Ljung

Author

\section{Sammanfattning}

Abstract

It is a typical situation in modern modeling that a total model is built up from simpler submodels, or modules, for example residing in a model library. The total model could be quite complex, while the modules are well understood and analysed. A procedure to decide global parameter identifiability for such a collection of model equations of differentialalgebraic nature is suggested. It is shown how to make use of the natural modularization of the model structure. Basically, global identifiability is obtained if and only if each module is identifiable, and the connecting signals can be retrieved from the external signals, without knowledge of the values of the parameters. 